\title{
Ising model across planar lattices
}

\author{
R.P.K.C. Malmini Ranasinghe \\ Department of Mathematics, Faculty of Applied Sciences, University of Sri Jayewardenepura, Gangodawila, Nugegoda.
}

\begin{abstract}
The Ising model on a two dimensional lattice with pentagon tiling and greater number of next to neighbours interaction is equivalent to the Ising model on the centered square lattice with nearest and next to nearest neighbour non-crossing interactions. We obtain exact expressions on calculation of critical temperature with ferromagnetic and anti-ferromagnetic interactions. A direct anti-ferromagnetic bond of strength $\alpha L$ can give rise to the transition temperatures for appropriate $\alpha$. We apply the star-triangle mapping transformation technique and decorating transform to Ising model.
\end{abstract}

Keywords: Exact results, Ising model, square lattice, transformations.

\section{INTRODUCTION}

Exact solution of the planar Ising model on a square lattice with nearest neighbour interactions were obtained in $1944^{1}$. Exact solutions for a number of more complex Lattices $^{2-5}$ and exact solution of the Ising model on the 4-6 lattice triangle, pentagonal and hexagonal lattices were obtained $^{6,7}$. Some attention has been made to the model on hierarchical lattices ${ }^{8}$. It is known, in fact, that all planar Ising models with non-crossing bonds are, in principle, solvable. However, it is still of interest to search for other exactly solved models towards particular interactions and to compute the critical point for those particular lattices with ferromagnetic and anti-ferromagnetic interactions. For this, various transformation methods like duality, startriangle and decoration-iteration to the Ising model on

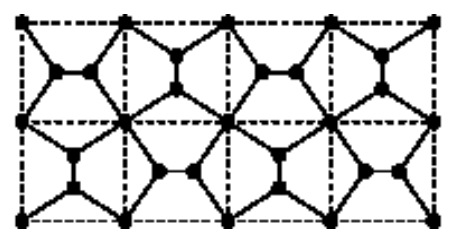

Figure 1: Pentagonal lattice with nearest neighbour interactions regular two-dimensional lattices have been investigated in the past ${ }^{9}, 10$. We consider a model with competing ferromagnetic and anti-ferromagnetic interactions acting on a two-dimensional lattice with pentagon tiling.

We consider an Ising model on a planar lattice where tiling is achieved by polygons, namely triangles, pentagons and septogons. The lattice view as a model by turning $90^{\circ}$ in the neighbouring square plaquette.

Inner spins in every plaquette of the pentagon lattice are replaced with triangles of spins linked between themselves at one identical site and additionally connected with their remaining sites with a pair of neighbouring corners of the square lattice. We have a lattice that is exactly mapped onto a pentagonal lattice that is illustrated in Figure 2.

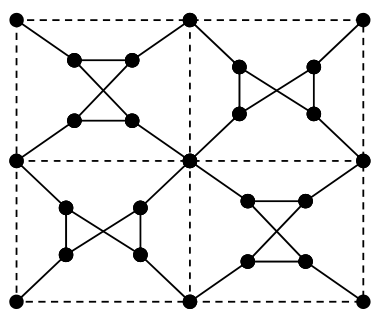

Figure 2: Star-triangle transformation for pentagonal lattice

Ordering the squares is in the same manner. Applying the transformation method to the lattice we have with triangles and septogones, we get the same situation.

There are two different interaction parameters $J$ and $J^{\prime}$ coupling particular nearest neighbour pairs of spins. Let $s_{1}, s_{2}, s_{3}$ and $s_{4}$ be corner spins located at the sites of a lattice and $\sigma_{1}$ and $\sigma_{2}$ be inner spins. The Ising Hamiltonian defined upon the underlying elementary plaquette is given by: 


$$
H=-J\left[\sigma_{1}\left(s_{1}+s_{2}\right)+\sigma_{2}\left(s_{3}+s_{4}\right)\right]-J^{\prime} \sigma_{1} \sigma_{2}
$$

Additional spin $s$ is introduced at each internal bond.

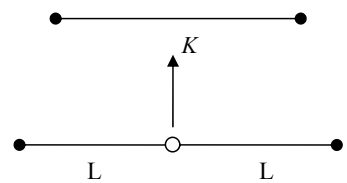

Figure 3: Effective coupling $k$

The basic decoration-iteration transform is shown in Figure 3, where summing over the central spin $s$ with coupling $L$ gives rise to a new effective coupling $K$ between the primary vertex spins $\sigma_{1}, \sigma_{2}$ :

$\sum_{s} \exp \left[L s\left(\sigma_{1}+\sigma_{2}\right)\right]=A \exp \left(K \sigma_{1} \sigma_{2}\right)$

Here $\sigma_{1}, \sigma_{2}= \pm 1, A=e^{-k^{\prime} / 2}$. The effective coupling parameter $\mathrm{k}^{\prime}=\beta J^{\prime}, \beta=\frac{1}{k_{\beta} T} . K_{\beta}$ is the Boltman's Constant and $\mathrm{T}$ is the absolute temperature and we get $A^{2}=4 \cosh (2 L)$.

The effective interaction between the spins $s$ and $\sigma_{1}, \sigma_{2}$ is defined by

$K=\frac{1}{2} \log [\cosh (2 L)]$

This is limit to the case $J^{\prime} / 2 \beta T>0$.

Summation in the partition function over the internal spins $\sigma_{1}, \sigma_{2}$ is the stat-triangle transformation and effective interaction follows the expression

$\sum_{\sigma_{1} \sigma_{2}} e^{L \sigma+k /\left(s_{1}+s_{2}\right) \sigma_{1}}=2 \cosh \left[L \sigma+\beta J\left(s_{1}+s_{2}\right)\right]=\beta e^{k_{1}\left(s_{1}+s_{2}\right) \sigma+k_{2} s_{1} s_{2}}$

$k=\beta J, \sigma= \pm 1, s_{1}= \pm 1, s_{2}= \pm 1$

We get an Ising model on the centered square lattice with the nearest-neighbours interaction $k_{1}$ and non-crossing diagonal interaction $k_{2}$.

The isotropic nearest-neighbours interaction

$$
k_{1}=\frac{1}{4}\left[\frac{\cosh \left(L+2 k^{\prime}\right)}{\cosh \left(L-2 k^{\prime}\right)}\right]
$$

and the non-crossing diagonal interaction between next to nearest-neighbours

$k_{2}=\frac{1}{4}\left[\frac{\cosh 2 L+\cos 4 k^{\prime}}{\cosh (2 L+1)}\right]$

We introduce one decorating spin as well as the direct anti-ferromagnetic bond. If a direct anti-ferromagnetic bond is stirred into the mix as well for good measure this becomes

$K=-\alpha L+\frac{1}{2} \log [\cosh (2 L)]$

In this case summing over the intermediate spins gives

$K=-\alpha L+\frac{1}{2} \log \left[\frac{(\exp (2 L)+1)^{2}+(\exp (2 L)-1)^{2}}{(\exp (2 L)+1)^{2}-(\exp (2 L)-1)^{2}}\right]$

along with an equation for the normalization factor

$A^{2}=4 \frac{\left[\sinh (2 L)^{2}\right]}{\sinh (2 K)}$

Ising spins, which distribute on a plane decorated lattice and couple with their neighbouring spins by antiferromagnetic exchange interaction, are found to show a number of varieties in its magnetic properties ${ }^{10}$. Basically, the form of the transformation in both equations (3) and (4) meant that three transitions could occur for an Ising model.

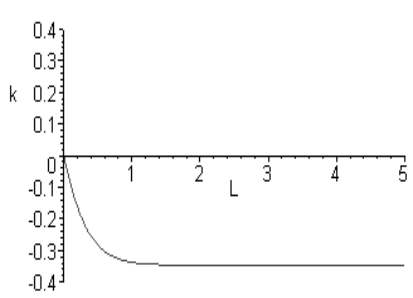

Figure 4: (a) $\mathrm{k}$ is $\approx-0.38$ when $\alpha \rightarrow 1$

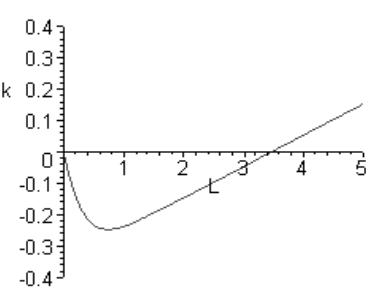

Figure 4: (b) $\alpha$ increased
For the square lattice Ising model for an individually decorated model, the minimum value of $K$ is $\approx-0.318$ when $\alpha \rightarrow l$ as in Figure 4(a). As $\alpha$ increased further $K(L)$ eventually becomes monotonically increasing function illustrated in Figure 4(b) where ferromagnetic transition disappears.

\section{DISCUSSION}

In conclusion, the Ising model on a pentagonal lattice with nearest neighbour and next to nearest neighbour interactions are described by two parameters and is equivalent to the Ising model on the centered square lattice. Exact expressions were obtained to compute critical temperature when a model with competing ferromagnetic and anti-ferromagnetic interactions acting on a two-dimensional lattice with pentagon tiling. It is shown that lattice with a large number of nearest neighbour interaction parameters will also be exactly solvable. 


\section{References}

1. Onsagar L. (1994). Crystal statistics two dimensional model with an order-disorder transition. Physical Review 65: 117.

2. Baxter R.J. (1982). Exactly solved Models in Statistical Mechanics. Academic Press, New York.

3. Baxter R.J. \& Wu F.Y. (1973). Exact solution of an Ising model with three-spin interactions on a triangular lattice. Physical Review Letters 31:1294-1297.

4. Syozi I. (1972). Phase Transition and Critical Phenomena, Vol. 1 (Eds. C. Domb \& M.S. Green) Academic Press, New York.

5. Otimma J. \& Keppert M. (2002). Exact solution of the Ising model on a 4-6 lattice. Journal of Physics A:

\section{Mathematical General 35:219-224.}

6. Urumov V. J. (2002). Exact solution of the Ising model on a pentagonal lattice. Journal of Physics A: Mathematical General 35:7317.

7. Gonçalves L.L.(1985). Uniaxial anisotropy effects in the Ising model: an exactly soluble model Physica Scripta 32: 248.

8. Johnston D.A. \& Ranasinghe P.K.C.M. (2002). Decorating random quadrangulations. Journal of Physics A: Mathematical General 35: 81-93.

9. Janke W., Johnston D.A. \& Ranasinghe P.K.C. M. (2002). Information geometry of the ising model on planar random graphs. Physical Review E 66:056119.

10. Nakano H. (1968). Ordering in certain statistical systems of Ising spins. Progress of Theoretical Physics 39: 1121. 